\title{
Erratum to: Search for annihilating dark matter in the Sun with 3 years of IceCube data
}

\author{
IceCube Collaboration
}

M. G. Aartsen' ${ }^{2}$, M. Ackermann ${ }^{52}$, J. Adams ${ }^{16}$, J. A. Aguilar $^{12}$, M. Ahlers ${ }^{30}$, M. Ahrens ${ }^{42}$, D. Altmann ${ }^{24}$, K. Andeen ${ }^{32}$, T. Anderson ${ }^{48}$, I. Ansseau ${ }^{12}$, G. Anton ${ }^{24}$, M. Archinger ${ }^{31}$, C. Argüelles ${ }^{14}$, J. Auffenberg ${ }^{1}$, S. Axani ${ }^{14}$, X. Bai ${ }^{40}$, S. W. Barwick ${ }^{27}$, V. Baum ${ }^{31}$, R. Bay ${ }^{7}$, J. J. Beatty ${ }^{18,19}$, J. Becker Tjus ${ }^{10}$, K.-H. Becker ${ }^{51}$, S. BenZvi ${ }^{49}$, D. Berley ${ }^{17}$, E. Bernardini ${ }^{52}$, A. Bernhard ${ }^{34}$, D. Z. Besson $^{28}$, G. Binder ${ }^{7,8}$, D. Bindig ${ }^{51}$, M. Bissok ${ }^{1}$, E. Blaufuss ${ }^{17}$, S. Blot ${ }^{52}$, C. Bohm ${ }^{42}$, M. Börner ${ }^{21}$, F. Bos ${ }^{10}$, D. Bose ${ }^{44}$, S. Böser ${ }^{31}$, O. Botner ${ }^{50}$, J. Braun ${ }^{30}$, L. Brayeur ${ }^{13}$, H.-P. Bretz ${ }^{52}$, S. Bron ${ }^{25}$, A. Burgman ${ }^{50}$, T. Carver ${ }^{25}$, M. Casier ${ }^{13}$, E. Cheung ${ }^{17}$, D. Chirkin ${ }^{30}$, A. Christov ${ }^{25}$, K. Clark $^{45}$, L. Classen ${ }^{35}$, S. Coenders ${ }^{34}$, G. H. Collin ${ }^{14}$, J. M. Conrad ${ }^{14}$, D. F. Cowen ${ }^{47,48}$, R. Cross ${ }^{49}$, M. Day ${ }^{30}$, J. P. A. M. de André22 , C. De Clercq ${ }^{13}$, E. del Pino Rosendo ${ }^{31}$, H. Dembinski ${ }^{36}$, S. De Ridder ${ }^{26}$, P. Desiati ${ }^{30}$, K. D. de Vries ${ }^{13}$, G. de Wasseige ${ }^{13}$, M. de With' ${ }^{9}$, T. DeYoung ${ }^{22}$, J. C. Díaz-Vélez ${ }^{30}$, V. di Lorenzo ${ }^{31}$, H. Dujmovic ${ }^{44}$, J. P. Dumm ${ }^{42}$, M. Dunkman ${ }^{48}$, B. Eberhardt ${ }^{31}$, T. Ehrhardt ${ }^{31}$, B. Eichmann ${ }^{10}$, P. Eller ${ }^{48}$, S. Euler ${ }^{50}$, P. A. Evenson ${ }^{36}$, S. Fahey ${ }^{30}$, A. R. Fazely ${ }^{6}$, J. Feintzeig ${ }^{30}$, J. Felde ${ }^{17}$, K. Filimonov ${ }^{7}$, C. Finley ${ }^{42}$, S. Flis ${ }^{42}$, C.-C. Fösig ${ }^{31}$, A. Franckowiak ${ }^{52}$, E. Friedman ${ }^{17}$, T. Fuchs ${ }^{21}$, T. K. Gaisser ${ }^{36}$, J. Gallagher ${ }^{29}$, L. Gerhardt ${ }^{7,8}$, K. Ghorbani ${ }^{30}$, W. Giang ${ }^{23}$, L. Gladstone ${ }^{30}$, T. Glauch ${ }^{1}$, T. Glüsenkamp ${ }^{24}$, A. Goldschmidt ${ }^{8}$, J. G. Gonzalez ${ }^{36}$,

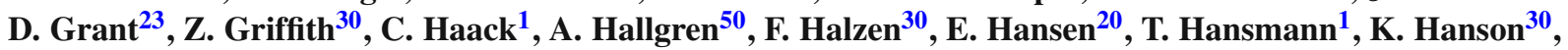

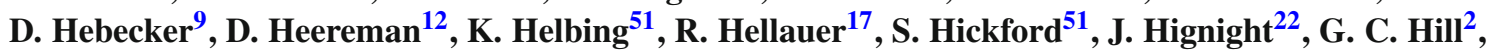
K. D. Hoffman ${ }^{17}$, R. Hoffmann ${ }^{51}$, K. Hoshina ${ }^{30,53}$, F. Huang ${ }^{48}$, M. Huber ${ }^{34}$, K. Hultqvist ${ }^{42}$, S. In ${ }^{44}$, A. Ishihara ${ }^{15}$, E. Jacobi ${ }^{52}$, G. S. Japaridze ${ }^{4}$, M. Jeong ${ }^{44}$, K. Jero ${ }^{30}$, B. J. P. Jones ${ }^{14}$, W. Kang ${ }^{44}$, A. Kappes ${ }^{35}$, T. Karg ${ }^{52}$, A. Karle ${ }^{30}$, U. Katz ${ }^{24}$, M. Kauer ${ }^{30}$, A. Keivani ${ }^{48}$, J. L. Kelley ${ }^{30}$, A. Kheirandish ${ }^{30}$, J. Kim ${ }^{44}$, M. Kim ${ }^{44}$, T. Kintscher ${ }^{52}$, J. Kiryluk ${ }^{43}$, T. Kittler ${ }^{24}$, S. R. Klein ${ }^{7,8}$, G. Kohnen ${ }^{33}$, R. Koirala ${ }^{36}$, H. Kolanoski ${ }^{9}$, R. Konietz ${ }^{1}$, L. Köpke ${ }^{31}$, C. $K_{0 p p e r}{ }^{23}$, S. Kopper ${ }^{11}$, D. J. Koskinen ${ }^{20}$, M. Kowalski ${ }^{9,52}$, K. Krings ${ }^{34}$, M. Kroll ${ }^{10}$, G. Krückl ${ }^{31}$, C. Krüger ${ }^{30}$, J. Kunnen ${ }^{13}$, S. Kunwar ${ }^{52}$, N. Kurahashi ${ }^{39}$, T. Kuwabara ${ }^{15}$, M. Labare ${ }^{26}$, J. L. Lanfranchi ${ }^{48}$, M. J. Larson ${ }^{20}$, F. Lauber ${ }^{51}$, D. Lennarz ${ }^{22}$, M. Lesiak-Bzdak ${ }^{43}$, M. Leuermann' ${ }^{1}$, L. Lu ${ }^{15}$, J. Lünemann ${ }^{13}$, J. Madsen ${ }^{41}$, G. Maggi ${ }^{13}$, K. B. M. Mahn ${ }^{22}$, S. Mancina ${ }^{30}$, M. Mandelartz ${ }^{10}$, R. Maruyama $^{37}$, K. Mase ${ }^{15}$, R. Maunu ${ }^{17}$, F. McNally ${ }^{30}$, K. Meagher ${ }^{12}$, M. Medici ${ }^{20}$, M. Meier ${ }^{21}$, A. Meli ${ }^{26}$, T. Menne ${ }^{21}$, G. Merino ${ }^{30}$, T. Meures ${ }^{12}$, S. Miarecki ${ }^{7,8}$, T. Montaruli ${ }^{25}$, M. Moulai ${ }^{14}$, R. Nahnhauer ${ }^{52}$, U. Naumann ${ }^{51}$, G. Neer ${ }^{22}$, H. Niederhausen ${ }^{43}$, S. C. Nowicki ${ }^{23}$, D. R. Nygren $^{8}$, A. Obertacke Pollmann ${ }^{51}$, A. Olivas ${ }^{17}$, A. O'Murchadha ${ }^{12}$, T. Palczewski ${ }^{7,8}$, H. Pandya ${ }^{36}$, D. V. Pankova ${ }^{48}$, P. Peiffer ${ }^{31}$, Ö. Penek ${ }^{1}$, J. A. Pepper ${ }^{46}$, C. Pérez de los Heros ${ }^{50}$, D. Pieloth ${ }^{21}$, E. Pinat ${ }^{12}$,

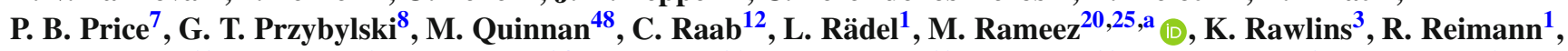
B. Relethford ${ }^{39}$, M. Relich ${ }^{15}$, E. Resconi ${ }^{34}$, W. Rhode ${ }^{21}$, M. Richman ${ }^{39}$, B. Riedel ${ }^{23}$, S. Robertson ${ }^{2}$, M. Rongen ${ }^{1}$, C. Rott $^{44}$, T. Ruhe ${ }^{21}$, D. Ryckbosch ${ }^{26}$, D. Rysewyk ${ }^{22}$, L. Sabbatini ${ }^{30}$, S. E. Sanchez Herrera ${ }^{23}$, A. Sandrock ${ }^{21}$, J. Sandroos $^{31}$, S. Sarkar ${ }^{20,38}$, K. Satalecka ${ }^{52}$, P. Schlunder ${ }^{21}$, T. Schmidt ${ }^{17}$, S. Schoenen ${ }^{1}$, S. Schöneberg ${ }^{10}$, L. Schumacher ${ }^{1}$, D. Seckel ${ }^{36}$, S. Seunarine ${ }^{41}$, D. Soldin ${ }^{51}$, M. Song ${ }^{17}$, G. M. Spiczak ${ }^{41}$, C. Spiering ${ }^{52}$, T. Stanev ${ }^{36}$, A. Stasik ${ }^{52}$, J. Stettner ${ }^{1}$, A. Steuer ${ }^{31}$, T. Stezelberger ${ }^{8}$, R. G. Stokstad ${ }^{8}$, A. Stöß1 ${ }^{15}$, R. Ström ${ }^{50}$, N. L. Strotjohann ${ }^{52}$, G. W. Sullivan ${ }^{17}$, M. Sutherland ${ }^{18}$, H. Taavola ${ }^{50}$, I. Taboada ${ }^{5}$, J. Tatar ${ }^{7,8}$, F. Tenholt ${ }^{10}$, S. Ter-Antonyan ${ }^{6}$,

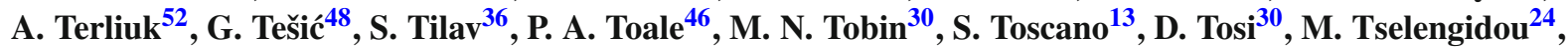
A. Turcati ${ }^{34}$, E. Unger ${ }^{50}$, M. Usner ${ }^{52}$, J. Vandenbroucke ${ }^{30}$, N. van Eijndhoven ${ }^{13}$, S. Vanheule ${ }^{26}$, M. van Rossem ${ }^{30}$, J. van Santen ${ }^{52}$, M. Vehring ${ }^{1}$, M. Voge ${ }^{11}$, E. Vogel ${ }^{1}$, M. Vraeghe ${ }^{26}$, C. Walck $^{42}$, A. Wallace ${ }^{2}$, M. Wallraff ${ }^{1}$, N. Wandkowsky ${ }^{30}$, Ch. Weaver ${ }^{23}$, M. J. Weiss ${ }^{48}$, C. Wendt $^{30}$, S. Westerhoff ${ }^{30}$, B. J. Whelan ${ }^{2}$, S. Wickmann ${ }^{1}$, K. Wiebe ${ }^{31}$, C. H. Wiebusch ${ }^{1}$, L. Wille ${ }^{30}$, D. R. Williams ${ }^{46}$, L. Wills ${ }^{39}$, M. Wolf ${ }^{42}$, T. R. Wood $^{23}$, E. Woolsey ${ }^{23}$, K. Woschnagg ${ }^{7}$, D. L. Xu ${ }^{30}$, X. W. $\mathrm{Xu}^{6}$, Y. Xu ${ }^{43}$, J. P. Yanez ${ }^{23}$, G. Yodh ${ }^{27}$, S. Yoshida' ${ }^{15}$, M. Zoll ${ }^{42, b}$

\footnotetext{
${ }^{1}$ III. Physikalisches Institut, RWTH Aachen University, 52056 Aachen, Germany

2 Department of Physics, University of Adelaide, Adelaide 5005, Australia
} 
${ }^{3}$ Department of Physics and Astronomy, University of Alaska Anchorage, 3211 Providence Dr., Anchorage, AK 99508, USA

${ }^{4}$ CTSPS, Clark-Atlanta University, Atlanta, GA 30314, USA

${ }^{5}$ School of Physics and Center for Relativistic Astrophysics, Georgia Institute of Technology, Atlanta, GA 30332, USA

${ }^{6}$ Department of Physics, Southern University, Baton Rouge, LA 70813, USA

${ }^{7}$ Department of Physics, University of California, Berkeley, CA 94720, USA

${ }^{8}$ Lawrence Berkeley National Laboratory, Berkeley, CA 94720, USA

${ }^{9}$ Institut für Physik, Humboldt-Universität zu Berlin, 12489 Berlin, Germany

${ }^{10}$ Fakultät für Physik \& Astronomie, Ruhr-Universität Bochum, 44780 Bochum, Germany

${ }^{11}$ Physikalisches Institut, Universität Bonn, Nussallee 12, 53115 Bonn, Germany

12 Science Faculty CP230, Université Libre de Bruxelles, 1050 Brussels, Belgium

${ }^{13}$ Dienst ELEM, Vrije Universiteit Brussel (VUB), 1050 Brussels, Belgium

${ }^{14}$ Department of Physics, Massachusetts Institute of Technology, Cambridge, MA 02139, USA

15 Department of Physics and Institute for Global Prominent Research, Chiba University, Chiba 263-8522, Japan

16 Department of Physics and Astronomy, University of Canterbury, Private Bag 4800, Christchurch, New Zealand

17 Department of Physics, University of Maryland, College Park, MD 20742, USA

${ }^{18}$ Department of Physics and Center for Cosmology and Astro-Particle Physics, Ohio State University, Columbus, OH 43210, USA

${ }^{19}$ Department of Astronomy, Ohio State University, Columbus, OH 43210, USA

20 Present Address: Niels Bohr Institute, University of Copenhagen, 2100 Copenhagen, Denmark

${ }^{21}$ Department of Physics, TU Dortmund University, 44221 Dortmund, Germany

22 Department of Physics and Astronomy, Michigan State University, East Lansing, MI 48824, USA

${ }^{23}$ Department of Physics, University of Alberta, Edmonton, AB T6G 2E1, Canada

${ }^{24}$ Erlangen Centre for Astroparticle Physics, Friedrich-Alexander-Universität Erlangen-Nürnberg, 91058 Erlangen, Germany

25 Département de physique nucléaire et corpusculaire, Université de Genève, 1211 Geneva, Switzerland

${ }^{26}$ Department of Physics and Astronomy, University of Gent, 9000 Ghent, Belgium

${ }^{27}$ Department of Physics and Astronomy, University of California, Irvine, CA 92697, USA

28 Department of Physics and Astronomy, University of Kansas, Lawrence, KS 66045, USA

${ }^{29}$ Department of Astronomy, University of Wisconsin, Madison, WI 53706, USA

${ }^{30}$ Department of Physics and Wisconsin IceCube Particle Astrophysics Center, University of Wisconsin, Madison, WI 53706, USA

${ }^{31}$ Institute of Physics, University of Mainz, Staudinger Weg 7, 55099 Mainz, Germany

32 Department of Physics, Marquette University, Milwaukee, WI 53201, USA

${ }^{33}$ Université de Mons, 7000 Mons, Belgium

${ }^{34}$ Physik-department, Technische Universität München, 85748 Garching, Germany

${ }^{35}$ Institut für Kernphysik, Westfälische Wilhelms-Universität Münster, 48149 Münster, Germany

36 Department of Physics and Astronomy, Bartol Research Institute, University of Delaware, Newark, DE 19716, USA

${ }^{37}$ Department of Physics, Yale University, New Haven, CT 06520, USA

38 Department of Physics, University of Oxford, 1 Keble Road, Oxford OX1 3NP, UK

${ }^{39}$ Department of Physics, Drexel University, 3141 Chestnut Street, Philadelphia, PA 19104, USA

${ }^{40}$ Physics Department, South Dakota School of Mines and Technology, Rapid City, SD 57701, USA

${ }^{41}$ Department of Physics, University of Wisconsin, River Falls, WI 54022, USA

${ }^{42}$ Department of Physics, Oskar Klein Centre, Stockholm University, 10691 Stockholm, Sweden

43 Department of Physics and Astronomy, Stony Brook University, Stony Brook, NY 11794-3800, USA

${ }^{44}$ Department of Physics, Sungkyunkwan University, Suwon 440-746, Korea

45 Department of Physics, University of Toronto, Toronto, ON M5S 1A7, Canada

46 Department of Physics and Astronomy, University of Alabama, Tuscaloosa, AL 35487, USA

${ }^{47}$ Department of Astronomy and Astrophysics, Pennsylvania State University, University Park, PA 16802, USA

48 Department of Physics, Pennsylvania State University, University Park, PA 16802, USA

49 Department of Physics and Astronomy, University of Rochester, Rochester, NY 14627, USA

50 Department of Physics and Astronomy, Uppsala University, Box 516, 75120 Uppsala, Sweden

${ }^{51}$ Department of Physics, University of Wuppertal, 42119 Wuppertal, Germany

52 DESY, 15735 Zeuthen, Germany

${ }^{53}$ Earthquake Research Institute, University of Tokyo, Bunkyo, Tokyo 113-0032, Japan

Received: 29 January 2019 / Accepted: 19 February 2019 / Published online: 8 March 2019

(C) The Author(s) 2019

Erratum to: Eur. Phys. J. C (2017) 77:146 https://doi.org/10.1140/epjc/s10052-017-4689-9

The original article can be found online at https://doi.org/10.1140/ epjc/s10052-017-4689-9.

a e-mail: mohamed.rameez@nbi.ku.dk

b e-mail: marcel.zoll.physics@ gmail.com
In the analysis published in Ref. [1], constraints on the number of signal events $n_{\mathrm{s}}$ can be interpreted as constraints on the volumetric neutrino to muon conversion rate $\Lambda_{\nu \bar{v} \rightarrow \mu^{+} \mu^{-}}$, 


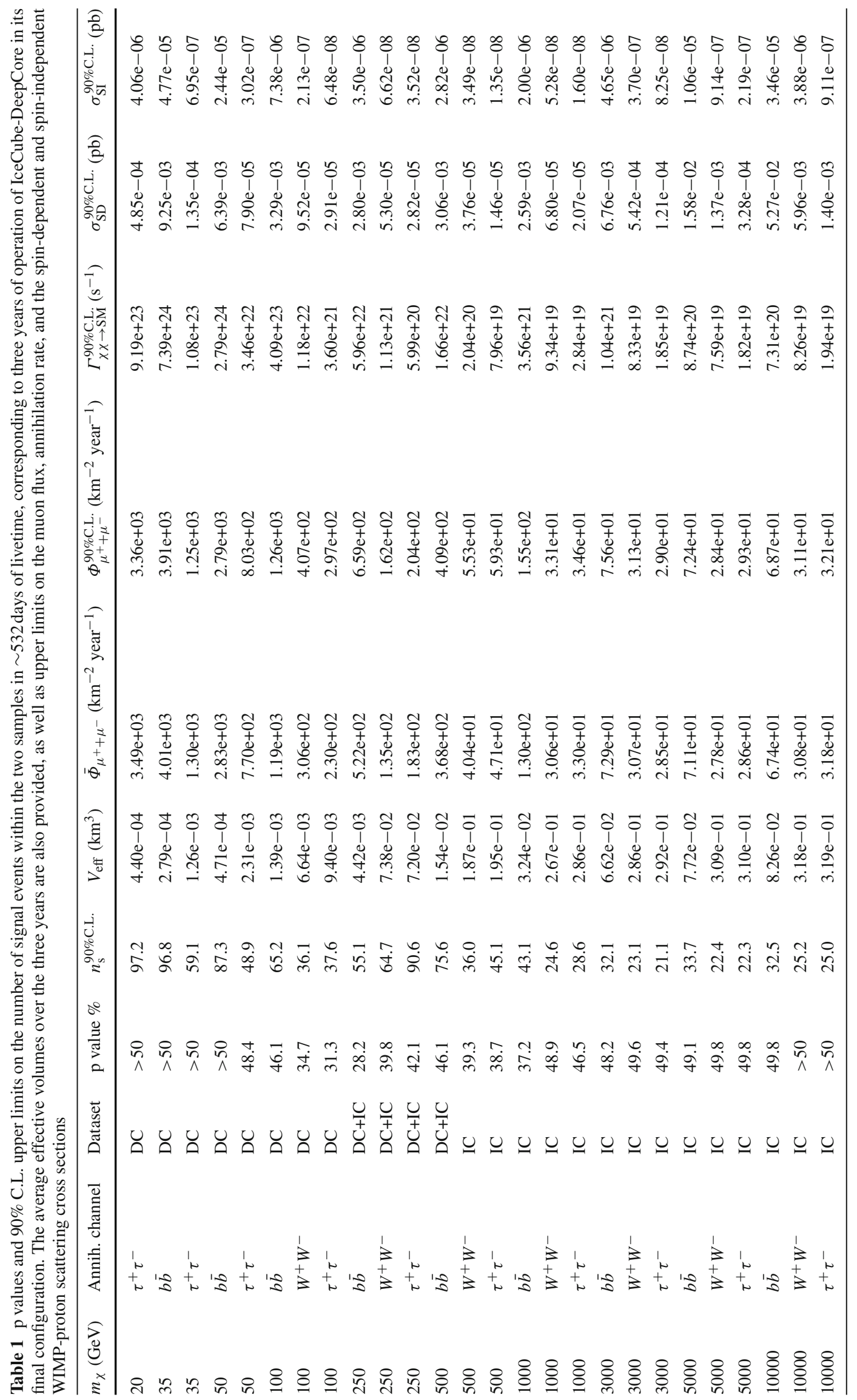


$\Lambda_{\nu \bar{v} \rightarrow \mu^{+} \mu^{-}}^{90 \% \text { C.L. }}=\frac{n_{\mathrm{s}}^{90 \% \text { C.L. }}}{\sum_{j} T_{\text {live }}^{j} V_{\text {eff }}^{j}}$,

where $T_{\text {live }}$ and $V_{\text {eff }}$ are the livetime and effective volume of the data sample of index $j$. These can then be interpreted as constraints on the muon flux $\Phi_{\mu^{+}+\mu^{-}}$, dark matter (DM) annihilation rate in the Sun $\Gamma_{\chi \chi} \rightarrow \mathrm{SM}$, as well as the spindependent (SD) and spin-independent (SI) scattering cross sections $\sigma_{\mathrm{SD}}$ and $\sigma_{\mathrm{SI}}$ using WimpSim [2].

In Table 4 of Ref. [1], the labels and units of columns 7 and 8 suggest that the muon flux $\Phi_{\mu^{+}+\mu^{-}}$(in units $\mathrm{km}^{-2}$ year $^{-1}$ ) is being presented. However for the first 12 rows, corresponding to points in which the DeepCore (DC) dataset was included, the volumetric neutrino to muon conversion rate $\Lambda_{\nu \bar{v} \rightarrow \mu^{+} \mu^{-}}$(in units $\mathrm{km}^{-3}$ year ${ }^{-1}$ ) were erroneously reported instead. The corrected table (Table 1) is presented hereby. All other columns remain unchanged. All quantities that go into the right hand side of Eq. 1 are presented in the table, as well as median sensitivities and $90 \%$ C.L. upper limits on the muon flux $\Phi_{\mu^{+}+\mu^{-}}$derived using WimpSim [2].

The final results and conclusions presented in Ref. [1] in terms of constraints on the SD and SI scattering cross sections $\sigma_{\mathrm{SD}}$ and $\sigma_{\mathrm{SI}}$ as well as the DM annihilation rate in the Sun $\Gamma_{\chi \chi} \rightarrow \mathrm{SM}$, remain unchanged.

In Section 4.2 of Ref. [1], the maximum zenith angle of the Sun is erroneously mentioned as $104^{\circ}$. The correct maximum zenith angle of the Sun is $114^{\circ}$ at the South Pole.

Acknowledgements We thank Joakim Edsjö and Tom Gaisser for the careful scrutiny through which these errors were brought to light.

The authors gratefully acknowledge support from the following agencies and institutions: USA - U.S. National Science Foundation-Office of Polar Programs, U.S. National Science Foundation-Physics Division, Wisconsin Alumni Research Foundation, Center for High Throughput Computing (CHTC) at the University of Wisconsin-Madison,
Open Science Grid (OSG), Extreme Science and Engineering Discovery Environment (XSEDE), U.S. Department of Energy-National Energy Research Scientific Computing Center, Particle astrophysics research computing center at the University of Maryland, Institute for Cyber-Enabled Research at Michigan State University, and Astroparticle physics computational facility at Marquette University; Belgium - Funds for Scientific Research (FRS-FNRS and FWO), FWO Odysseus and Big Science programmes, and Belgian Federal Science Policy Office (Belspo); Germany - Bundesministerium für Bildung und Forschung (BMBF), Deutsche Forschungsgemeinschaft (DFG), Helmholtz Alliance for Astroparticle Physics (HAP), Initiative and Networking Fund of the Helmholtz Association, Deutsches Elektronen Synchrotron (DESY), and High Performance Computing cluster of the RWTH Aachen; Sweden - Swedish Research Council, Swedish Polar Research Secretariat, Swedish National Infrastructure for Computing (SNIC), and Knut and Alice Wallenberg Foundation; Australia - Australian Research Council; Canada - Natural Sciences and Engineering Research Council of Canada, Calcul Québec, Compute Ontario, Canada Foundation for Innovation, WestGrid, and Compute Canada; Denmark Villum Fonden, Danish National Research Foundation (DNRF), Carlsberg Foundation; New Zealand - Marsden Fund; Japan - Japan Society for Promotion of Science (JSPS) and Institute for Global Prominent Research (IGPR) of Chiba University; Korea - National Research Foundation of Korea (NRF); Switzerland - Swiss National Science Foundation (SNSF).

Open Access This article is distributed under the terms of the Creative Commons Attribution 4.0 International License (http://creativecomm ons.org/licenses/by/4.0/), which permits unrestricted use, distribution, and reproduction in any medium, provided you give appropriate credit to the original author(s) and the source, provide a link to the Creative Commons license, and indicate if changes were made.

Funded by SCOAP ${ }^{3}$.

\section{References}

1. M.G. Aartsen et al. (IceCube Collaboration), Eur. Phys. J. C 77, 146 (2017)

2. M. Blennow, J. Edsjö, T. Ohlsson, JCAP 01, 021 (2008) 